\title{
An elementary approach to the inviscid limits for the 3D Navier-Stokes equations with slip boundary conditions and applications to the 3D Boussinesq equations
}

\author{
Luigi C. Berselli and Stefano Spirito
}

\begin{abstract}
In this note we consider the inviscid limit for the 3D Boussinesq equations without diffusion, under slip boundary conditions of Navier's type. We first study more closely the Navier-Stokes equations, to better understand the problem. The role of the initial data is also emphasized in connection with the vanishing viscosity limit.
\end{abstract}

Mathematics Subject Classification (1991). 35Q30, 35B25, 35B30, 76D05.

Keywords. Boussinesq equations, Navier-Stokes equations,

Vanishing viscosity limits.

\section{Introduction}

The aim of this note is to study the $L^{\infty}\left(0, T ; L^{2}(\Omega)\right)$ convergence, as $\nu$ vanishes, of (Leray-Hopf) weak solutions to the 3D Navier-Stokes equations (NSE), towards smooth solutions of the 3D Euler equations. The dependence of the rate of convergence in terms of different hypotheses on the initial data is studied and the results are also applied to deal with the problem of convergence of solution of the 3D Boussinesq equations to those of the Euler-Boussinesq.

We first study the problem with constant density (set for simplicity equal to one) and then, in the final section, we treat the Boussinesq equations. In particular, we start by considering the inviscid limit for the NSE in a bounded domain $\Omega \subset \mathbb{R}^{3}$ with smooth boundary $\Gamma:=\partial \Omega \neq \emptyset$.

For the reader's convenience we recall that when $\Gamma$ is non empty, for the NSE with Dirichlet boundary conditions

$$
\begin{array}{ll}
\partial_{t} u^{\nu}-\nu \Delta u^{\nu}+\left(u^{\nu} \cdot \nabla\right) u^{\nu}+\nabla p^{\nu}=0 & \text { in } \Omega \times(0, T], \\
\nabla \cdot u^{\nu}=0 & \text { in } \Omega \times(0, T], \\
u^{\nu}=0 & \text { on } \Gamma \times(0, T], \\
u^{\nu}(0, x)=u_{0}^{\nu} & \text { in } \Omega,
\end{array}
$$


in general one cannot have convergence (even in weak norms) towards smooth solutions of the Euler equations, even with the same initial data $u_{0}^{\nu}=u_{0}^{E}$

$$
\begin{array}{ll}
\partial_{t} u^{E}+\left(u^{E} \cdot \nabla\right) u^{E}+\nabla p^{E}=0 & \text { in } \Omega \times(0, T], \\
\nabla \cdot u^{E}=0 & \text { in } \Omega \times(0, T], \\
u^{E} \cdot n=0 & \text { on } \Gamma \times(0, T], \\
u^{E}(0, x)=u_{0}^{E} & \text { in } \Omega,
\end{array}
$$

see e.g. the review in Constantin [9] and Mazzucato [23]. In fact, even if both $u^{E}$ and $u^{\nu}$ are very smooth and both exist in $[0, T]$ (for some positive $T$ independent of the viscosity) certain extra-assumptions are needed in order to show, at least, that

$$
\text { as } \nu \rightarrow 0 \quad u^{\nu}(t) \rightarrow u^{E}(t) \quad \text { in } L^{2}(\Omega), \quad \text { uniformly in } t \in[0, T] .
$$

Some necessary and sufficient conditions, related with the dissipation of energy in a boundary-strip of width depending on $\nu$, have been detected by Kato [14]. See also recent developments in Temam and Wang [27], Wang [28], and Kelliher [15]. The lack of convergence is due to the boundary layer created from the difference between the tangential velocity of the Navier-Stokes solution and that of the Euler solution at the boundary: the first vanishes, while we do not have control on the tangential velocity of the Euler equations.

Better results can be obtained in the case of the NSE with Navier's boundary conditions. In Iftimie and Planas [12] it is considered the following initial-boundary value problem

$$
\begin{array}{ll}
\partial_{t} u^{\nu}-\nu \Delta u^{\nu}+\left(u^{\nu} \cdot \nabla\right) u^{\nu}+\nabla p^{\nu}=0 & \text { in } \Omega \times(0, T], \\
\nabla \cdot u^{\nu}=0 & \text { in } \Omega \times(0, T], \\
u^{\nu} \cdot n=0 & \text { on } \Gamma \times(0, T], \\
{\left[\mathcal{D}\left(u^{\nu}\right) n+\beta u^{\nu}\right]_{\tan }=0} & \text { on } \Gamma \times(0, T], \\
u^{\nu}(0, x)=u_{0}^{\nu} & \text { in } \Omega,
\end{array}
$$

where $\mathcal{D}\left(u^{\nu}\right)=\frac{1}{2}\left[\nabla u^{\nu}+\left(\nabla u^{\nu}\right)^{T}\right]$ is the deformation tensor, $\beta \geq 0$ is a constant (the friction coefficient) and $\left[\mathcal{D}\left(u^{\nu}\right) n+\beta u^{\nu}\right]_{\tan }$ is the tangential component of the vector $\mathcal{D}\left(u^{\nu}\right) n+\beta u^{\nu}$. This system is very close to that originally proposed by Navier [24] and studied analytically (in the stationary case) starting from Solonnikov and Ščadilov [25]. In particular, the Navier's slip conditions read as $\left[\nu \mathcal{D}\left(u^{\nu}\right) n+\beta u^{\nu}\right]_{\tan }=0$, hence authors in [12] are implicitly assuming the Maxwell scaling [22], with the friction parameter $\beta$ depending linearly on the viscosity. More details on the role of Navier's boundary conditions especially for numerical simulations, and some of the crucial differences between the two dimensional and three dimensional case, can be found in the review paper [7]. In the $2 \mathrm{D}$ setting the problem is slightly less-hard and classical results employing slip boundary conditions are those of Yudovich [34], Lions [17], and Bardos [1]. Interesting recent results in the $2 \mathrm{D}$ case are those in $[19,20]$.

A recent vanishing viscosity result in the $3 \mathrm{D}$ is the following one (see Theorem 1 in [12]). 
Theorem 1.1. Let $\Omega$ be a bounded smooth open set in $\mathbb{R}^{3}$ and let $u_{0} \in H^{3}(\Omega)$ a divergence-free vector field tangent to the boundary. For each $\nu>0$ consider $u_{0}^{\nu} \in L^{2}(\Omega)$ a divergence free vector field tangent to the boundary such that $u_{0}^{\nu} \rightarrow u_{0}$ strongly in $L^{2}(\Omega)$, as $\nu \rightarrow 0$. Let $u^{\nu}$ be a weak solution of the Navier-Stokes equations (1.3) with Navier's boundary conditions and with initial datum $u_{0}^{\nu}$. Let $u^{E} \in C\left([0, T] ; H^{3}(\Omega)\right)$ be the unique solution of the Euler equations (1.2), with initial datum $u_{0}$, for some $\left.T \in\right] 0, T_{\max }\left[\right.$, being $T_{\max }$ the maximal time of existence of the smooth solution of the Euler equations. Then, $u^{\nu}$ converges to $u^{E}$ strongly in $L^{\infty}\left(0, T ; L^{2}(\Omega)\right)$, as $\nu \rightarrow 0$.

Remark 1.2. By inspecting the proof, one can observe that, if the initial data converge in $L^{2}(\Omega)$ fast enough, then the same argument implies that $\sup _{t \in[0, T]}\left\|u^{\nu}(t)-u^{E}(t)\right\|^{2}=\mathcal{O}(\nu)$ and $\int_{0}^{T}\left\|\nabla u^{\nu}(\tau)-\nabla u^{E}(\tau)\right\|^{2} d \tau=\mathcal{O}(1)$. The result here is independent of the parameter $\beta \geq 0$.

Our aim is to study the convergence under some different slip-withoutfriction boundary conditions, involving the vorticity. More precisely we will study the following initial-boundary value problem

$$
\begin{array}{ll}
\partial_{t} u^{\nu}-\nu \Delta u^{\nu}+\left(u^{\nu} \cdot \nabla\right) u^{\nu}+\nabla p^{\nu}=0 & \text { in } \Omega \times(0, T], \\
\nabla \cdot u^{\nu}=0 & \text { in } \Omega \times(0, T], \\
u^{\nu} \cdot n=0 & \text { on } \Gamma \times(0, T], \\
\operatorname{curl} u^{\nu} \times n=0 & \text { on } \Gamma \times(0, T], \\
u^{\nu}(0, x)=u_{0}^{\nu} & \text { in } \Omega .
\end{array}
$$

and we will show how the convergence-rate can be improved.

The interest for these vorticity based Navier's boundary conditions is increasing, especially after the recent results by Xiao and Xin [31,32] and Beirão da Veiga and Crispo [3-6], and [8] concerning strong solutions and strong convergence. See also the related work by Xin et al. [29,30,33]. Next, we point out that the connection between the two Navier's type conditions, (1.3) versus (1.4), is expressed by the following identity, valid for all tangential vectors $\tau$ on the boundary $\Gamma$ :

$$
t \cdot \tau=\frac{\nu}{2}(\operatorname{curl} u \times n) \cdot \tau-\nu u \cdot \frac{\partial n}{\partial \tau} \quad \text { on } \Gamma .
$$

Here $t$ is the Cauchy stress vector defined by

$$
t(u, p):=n \cdot \mathbb{T}(u, p)=\sum_{k=1}^{n} \mathbb{T}_{i k}(u, p) n_{k},
$$

with $\mathbb{T}(u, p):=-\mathrm{I} p+\nu \mathcal{D}(u)$. This vector identity valid on $\Gamma$ shows that the two Navier's conditions are essentially the same in the case of a domain with flat boundary. Moreover, in a general domain they differ by a lower order term.

Results similar to the present ones have been also recently obtained by Xiao and Xin [32], while the results presented here are part of the Ph.D. thesis of the second author, completed at the end of 2011. We point out that the approach in [32] is slightly different, focusing more on $H^{1}(\Omega)$ convergence in the case of well-prepared initial datum $u_{0}^{\nu}=u_{0}^{E} \in H^{3}(\Omega)$ for NSE and 
Euler equations. In addition, the boundary value problem for the NavierStokes equations is a non-standard one, slightly different from (1.4). On the other hand, the aim of our result is not the study of the asymptotic limit of boundary layer, see $[13,21]$, in the case of vorticity based boundary conditions but is to show how the initial data affect the convergence of the vanishing viscosity even in the energy norm and to give a simple proof of the convergence in the case of well-prepared initial data. The main result of this paper is Theorem 3.2, which shows improved convergence in the $L^{2}$-norm and convergence also of first derivatives for the system (1.4), when the initial datum has vorticity vanishing at the boundary. Observe that in Theorem 3.1 the vanishing vorticity is requested only for the initial datum $u_{0}^{E}$ of the Euler equations. The initial data $u_{0}^{\nu}$ of the NSE are just divergence-free vector fields, tangential to the boundary, and converging merely in $L^{2}(\Omega)$ (hence without any control on the vorticity) to the datum of the Euler equations.

In the final section we use the results obtained for the NSE equations to tackle the following problem: we study the convergence of $\left(v^{\nu}, \rho^{\nu}\right)$, solution of the viscous Boussinesq equations

$$
\begin{array}{ll}
\partial_{t} v^{\nu}-\nu \Delta v^{\nu}+\left(v^{\nu} \cdot \nabla\right) v^{\nu}+\nabla q^{\nu}=-\rho^{\nu} e_{3} & \text { in } \Omega \times(0, T], \\
\partial_{t} \rho^{\nu}+\left(v^{\nu} \cdot \nabla\right) \rho^{\nu}=0 & \text { in } \Omega \times(0, T], \\
\nabla \cdot v^{\nu}=0 & \text { in } \Omega \times(0, T], \\
v^{\nu} \cdot n=0 & \text { on } \Gamma \times(0, T], \\
\operatorname{curl} v^{\nu} \times n=0 & \text { on } \Gamma \times(0, T], \\
v^{\nu}(0, x)=v_{0}^{\nu} & \text { in } \Omega, \\
\rho^{\nu}(0, x)=\rho_{0}^{\nu} & \text { in } \Omega,
\end{array}
$$

toward those of the Euler-Boussinesq equations

$$
\begin{array}{ll}
\partial_{t} v^{E}+\left(v^{E} \cdot \nabla\right) v^{E}+\nabla q^{E}=-\rho^{E} e_{3} & \text { in } \Omega \times(0, T], \\
\partial_{t} \rho^{E}+\left(v^{E} \cdot \nabla\right) \rho^{E}=0 & \text { in } \Omega \times(0, T], \\
\nabla \cdot v^{E}=0 & \text { in } \Omega \times(0, T], \\
v^{E} \cdot n=0 & \text { on } \Gamma \times(0, T], \\
v^{E}(0, x)=v_{0}^{E} & \text { in } \Omega, \\
\rho^{E}(0, x)=\rho_{0}^{E} & \text { in } \Omega,
\end{array}
$$

in the energy space $L^{\infty}\left(0, T ; L^{2}(\Omega)\right)$, where $e_{3}$ is the third vector of the canonical basis in $\mathbb{R}^{3}$.

Plan of the paper: In Sect. 2 we briefly recall the notation, some vector identities, and some existence results for the NSE (1.4). Next, in Sect. 3 we prove two different vanishing viscosity results for the NSE, showing the critical dependence on the initial datum. Finally, in Sect. 4 we study the vanishing viscosity limit for the Boussinesq system.

\section{Preliminaries}

We consider a bounded domain $\Omega \subset \mathbb{R}^{3}$ with smooth boundary $\Gamma$, say of class $C^{4}$, and $n$ denotes the exterior normal unit vector on $\Gamma$. We will use the classical Lebesgue spaces $\left(L^{2}(\Omega),\|\cdot\|\right)$ and $\left(L^{2}(\Gamma),\|\cdot\|_{\Gamma}\right)$ and the Sobolev 
spaces $\left(H^{k}(\Omega),\|\cdot\|_{k}\right)$ for $k \in \mathbb{N}$ (we do not distinguish between scalar and vector valued functions). We will denote by $\left(H^{s}(\Gamma),\|\cdot\|_{s, \Gamma}\right)$ the standard trace spaces on the boundary $\Gamma$. We will also denote by $C$ generic constants, which may change from line to line, but which are independent of the viscosity and of the solution of the equations we are considering.

We first start by recalling the precise notion of weak solution for the NSE we will use.

Definition 2.1. We say that $u^{\nu} \in L^{\infty}\left(0, T ; L^{2}(\Omega)\right) \cap L^{2}\left(0, T ; H^{1}(\Omega)\right)$, weakly divergence-free and tangential to the boundary, is a (Leray-Hopf) weak solution of the Navier-Stokes equations (1.4) if the two following condition hold:

$$
\begin{gathered}
\int_{0}^{T} \int_{\Omega}\left(-u^{\nu} \phi_{t}+\nu \nabla u^{\nu} \nabla \phi-\left(u^{\nu} \cdot \nabla\right) \phi u^{\nu}\right) d x d \tau \\
\quad+\nu \int_{0}^{T} \int_{\Gamma} u^{\nu} \cdot(\nabla n)^{T} \cdot \phi d S d \tau=\int_{\Omega} u_{0}^{\nu} \phi(0) d x
\end{gathered}
$$

for all vector-fields $\phi \in C_{0}^{\infty}([0, T[\times \bar{\Omega})$ such that $\nabla \cdot \phi=0$ in $\Omega \times[0, T[$, and $\phi \cdot n=0$ on $\Gamma \times[0, T[$. Moreover, the following energy estimate

$$
\begin{aligned}
& \frac{1}{2} \int_{\Omega}\left|u^{\nu}(t)\right|^{2} d x+\nu \int_{0}^{t} \int_{\Omega}\left|\nabla u^{\nu}\right|^{2} d x d \tau \\
& \quad+\nu \int_{0}^{t} \int_{\Gamma} u^{\nu} \cdot(\nabla n)^{T} \cdot u^{\nu} d S d \tau \leq \frac{1}{2} \int_{\Omega}\left|u_{0}^{\nu}\right|^{2} d x,
\end{aligned}
$$

is satisfied for all $t \in[0, T]$.

With this definition we have the following result.

Theorem 2.2. Let be given any positive $T>0$ and $u_{0}^{\nu} \in L^{2}(\Omega)$ which is weakly divergence-free and such that $u_{0}^{\nu} \cdot n=0$ on $\Gamma$. Then, there exists at least a weak solution $u^{\nu}$ of the Navier-Stokes equations (1.4) on $[0, T]$.

The proof of global existence of weak solution in the sense of the Definition 2.1 can be found for instance in $[31, \S 6]$. We observe now that our definition of energy inequality is slightly different from that in the above reference and we explain now the equivalence. To this end we recall the following formulas for integration by parts (see Ref. [2] for the proof).

Lemma 2.3. Let $u$ and $\phi$ be two smooth enough vector fields, tangential to the boundary $\Gamma$. Then it follows

$$
-\int_{\Omega} \Delta u \phi d x=\int_{\Omega} \nabla u \nabla \phi d x-\int_{\Gamma}(\omega \times n) \phi d S+\int_{\Gamma} u \cdot(\nabla n)^{T} \cdot \phi d S,
$$

where $\omega=\operatorname{curl} u$. Moreover, if $\nabla \cdot u=0$, then $-\Delta u=\operatorname{curl} \operatorname{curl} u$, and

$$
\int_{\Omega} \operatorname{curl} \omega \phi d x=-\int_{\Omega} \Delta u \phi d x=\int_{\Omega} \omega(\operatorname{curl} \phi) d x+\int_{\Gamma}(\omega \times n) \phi d S .
$$


Constructing weak solutions by the usual Galerkin method as in [31], we get for the approximate solutions $\left\{u_{m}^{\nu}\right\}_{m \in \mathbb{N}}$ the following identity:

$$
\frac{1}{2} \int_{\Omega}\left|u_{m}^{\nu}(t)\right|^{2} d x+\nu \int_{0}^{t} \int_{\Omega}\left|\operatorname{curl} u_{m}^{\nu}\right|^{2} d x d \tau=\frac{1}{2} \int_{\Omega}\left|u_{0 m}^{\nu}\right|^{2} d x \quad t \in[0, T],
$$

where we used as test function the function $u_{m}^{\nu}$ itself and the second integration by parts formula from Lemma 2.3 with $u=\phi=u_{m}^{\nu}$ (here the boundary integral vanishes due to the vorticity-based Navier's boundary conditions). Then, the usual compactness tools imply that $u_{m}^{\nu}$ converge as $m \rightarrow+\infty$ to a weak solution $u^{\nu}$ and the lower-semi-continuity of the norm implies that

$$
\frac{1}{2} \int_{\Omega}\left|u^{\nu}(t)\right|^{2} d x+\nu \int_{0}^{t} \int_{\Omega}\left|\operatorname{curl} u^{\nu}\right|^{2} d x d \tau \leq \frac{1}{2} \int_{\Omega}\left|u_{0}^{\nu}\right|^{2} d x
$$

which is the energy inequality (6.12) in [31]. Finally, by using the first integration by parts formula from Lemma 2.3 we get (2.2). This inequality will be used later on to make some of the calculations (which will be otherwise only formal) completely justified.

To conclude, we recall a well-known existence theorem for smooth solutions of the Euler equations (1.2) in Sobolev spaces.

Theorem 2.4. Let be given $u_{0}^{E} \in H^{3}(\Omega)$ such that $\nabla \cdot u_{0}^{E}=0$ and $u_{0}^{E} \cdot n=0$ on $\Gamma$. Then, there exists a positive time $T=T\left(\left\|u_{0}^{E}\right\|_{3}\right)>0$ such that a unique solution of (1.2) exists in

$$
u^{E} \in C\left([0, T] ; H^{3}(\Omega)\right) .
$$

The proof in the case of a bounded domain can be found in Ebin and Marsden [10] and Temam [26]. In particular $T \geq \frac{C}{\left\|u_{0}^{E}\right\|_{3}}$, for some $C>0$ independent of the solution and in the sequel $T$ will be any positive time strictly smaller than the maximal time of existence $T_{\max }$.

\section{Proof of the convergence results}

We start by showing the basic convergence result, which is the counterpart of $[12$, Thm. 1] in our setting.

Theorem 3.1. Let $\Omega$ be a bounded smooth open set in $\mathbb{R}^{3}$, and let $u_{0}^{E} \in$ $H^{3}(\Omega)$, be a divergence-free vector-field tangential to the boundary. Let $u^{E} \in$ $C\left([0, T] ; H^{3}(\Omega)\right)$ be the unique solution of the Euler equations (1.2), with initial datum $u_{0}$ and defined in some interval $[0, T]$. Let $u^{\nu}$ be a weak solution of the NSE (1.4) with divergence-free and tangential to the boundary initial datum $u_{0}^{\nu} \in L^{2}(\Omega)$, and with the vorticity-based Navier's conditions. Suppose also that

$$
\left\|u_{0}^{\nu}-u_{0}^{E}\right\|=\mathcal{O}\left(\nu^{\frac{3}{2}}\right) .
$$

Then,

$$
\sup _{t \in[0, T]}\left\|u^{\nu}(t)-u^{E}(t)\right\|^{2}=\mathcal{O}\left(\nu^{\frac{3}{2}}\right)
$$


and

$$
\int_{0}^{T}\left\|\nabla u^{\nu}(\tau)-\nabla u^{E}(\tau)\right\|^{2} d \tau=\mathcal{O}\left(\nu^{\frac{1}{2}}\right) .
$$

Proof. The proof is simply obtained by taking the difference of the equation satisfied by $u^{\nu}$ with that for $u^{E}$, multiplying by $u:=u^{\nu}-u^{E}$, and integrating by parts over $\Omega \times(0, T)$. Unfortunately, this cannot be done in a so straightforward manner since $u^{\nu}$ is a weak solution, hence using directly $u$ (having the regularity of $u^{\nu}$ ) as test function is not allowed. We need to pass to an integral formulation and to use the energy inequality (2.2) to make the argument rigorous. The reader well-acquainted with the argument can go directly to the formula (3.6).

We first observe that since $u^{E}$ is a smooth solutions of the Euler equations (1.2) in $[0, T] \times \Omega, u^{E}$ is allowed as test function for the NSE. Then, after certain integration by parts, we get for all $t \in[0, T]$

$$
\begin{aligned}
& \int_{\Omega} u^{\nu}(t) u^{E}(t) d x+\int_{0}^{t} \int_{\Omega}\left(\nu \nabla u^{\nu} \nabla u^{E}-u^{\nu} u_{t}^{E}+\left(u^{\nu} \cdot \nabla\right) u^{\nu} u^{E}\right) d x d \tau \\
& \quad+\nu \int_{0}^{t} \int_{\Gamma} u^{\nu}(\nabla n)^{T} u^{E} d S d \tau=\int_{\Omega} u_{0}^{E} u_{0}^{\nu} d x
\end{aligned}
$$

A further identity is obtained by multiplying the Euler equations by $u^{\nu}$. Since $u^{E}$ is a local smooth solution everything is well-defined and we get

$$
\int_{0}^{t} \int_{\Omega}\left(u_{t}^{E} u^{\nu}+\left(u^{E} \cdot \nabla\right) u^{E} u^{\nu}\right) d x d \tau=\int_{\Omega} u_{0}^{E} u_{0}^{\nu} d x .
$$

Next, by multiplying the Euler equations by $u^{E}$ and by the usual integrations by parts we get the energy conservation

$$
\frac{1}{2} \int_{\Omega}\left|u^{E}(t)\right|^{2} d x=\frac{1}{2} \int_{\Omega}\left|u_{0}^{E}\right|^{2} d x
$$

where we used the fact that $u^{E}$ is smooth, tangential to the boundary, and divergence-free.

Then, by adding together (2.2)-(3.3) and subtracting (3.1)-(3.2), we get

$$
\begin{aligned}
& \frac{\|u(t)\|^{2}}{2}+\nu \int_{0}^{t} \int_{\Omega} \nabla u^{\nu} \nabla u d x d \tau+\int_{0}^{t} \int_{\Omega}(u \cdot \nabla) u^{E} u d x d \tau \\
& +\nu \int_{0}^{t} \int_{\Gamma} u^{\nu}(\nabla n)^{T} u d S d \tau \leq \frac{\|u(0)\|^{2}}{2} .
\end{aligned}
$$

Let us consider the second term from the left-hand side of (3.4). By using the parallelogram equality we get

$$
\begin{aligned}
\int_{0}^{t} \int_{\Omega} \nabla u^{\nu} \nabla u d x d \tau= & \frac{1}{2} \int_{0}^{t} \int_{\Omega}\left|\nabla u^{\nu}\right|^{2} d x d \tau+\frac{1}{2} \int_{0}^{t} \int_{\Omega}|\nabla u|^{2} d x d \tau \\
& -\frac{1}{2} \int_{0}^{t} \int_{\Omega}\left|\nabla u^{E}\right|^{2} d x d \tau
\end{aligned}
$$


Then, we estimate the other two terms from the left-hand side of (3.4). We handle the third term by using the higher-order regularity of $u^{E}$, namely we use $u^{E} \in C\left([0, T] ; H^{3}(\Omega)\right) \hookrightarrow C\left([0, T] ; W^{1, \infty}(\Omega)\right)$, to get

$$
\left|\int_{0}^{t} \int_{\Omega}(u \cdot \nabla) u^{E} u d x d \tau\right| \leq C \int_{0}^{t}\|u(t)\|^{2} d \tau
$$

for some $C$ independent of $\nu$. Concerning the boundary term in (3.4), by using trace theorems and Young inequality we get, due to the smoothness of $\Gamma$,

$$
\begin{aligned}
\left|\nu \int_{0}^{t} \int_{\Gamma} u^{\nu}(\nabla n)^{T} u d S d \tau\right| & \leq \nu \int_{\Gamma}\left|u^{\nu}\right||\nabla n||u| d S \\
& \leq C \nu\left\|u^{\nu}\right\|_{\Gamma}\|u\|_{\Gamma} \\
& \leq C \nu\left\|u^{\nu}\right\|^{\frac{1}{2}}\left\|\nabla u^{\nu}\right\|^{\frac{1}{2}}\|u\|^{\frac{1}{2}}\|\nabla u\|^{\frac{1}{2}} \\
& \leq \frac{\nu}{4}\left\|\nabla u^{\nu}\right\|^{2}+\frac{\nu}{4}\|\nabla u\|^{2}+C \nu\left\|u^{\nu}\right\|\|u\| .
\end{aligned}
$$

In particular, to remove the zero-order term we have used the fact that for functions tangential to the boundary the Poincaré inequality holds true (see for instance, Kozono and Yanagisawa [16]) and also that from the energy inequality both $\left\|u^{\nu}\right\|$ and $u^{E}$ are bounded.

By collecting all the estimates, from (3.4) we get that

$$
\|u(t)\|^{2}+\nu \int_{0}^{t}\|\nabla u(\tau)\|^{2} d \tau \leq\|u(0)\|^{2}+C\left[\int_{0}^{t}\|u(\tau)\|^{2} d \tau+\nu\right] .
$$

We can now use Gronwall lemma, obtaining

$$
\left\|u^{\nu}-u^{E}\right\|_{L^{\infty}\left(0, T ; L^{2}(\Omega)\right)}^{2}=O(\nu) \quad \text { and } \quad\left\|\nabla u^{\nu}-\nabla u^{E}\right\|_{L^{2}\left(0, T ; L^{2}(\Omega)\right)}^{2}=O(1),
$$

exactly as in [12]. This is not the result stated in the Theorem 3.1, but the calculations are given to better understand the differences/improvement.

We make now some slightly different manipulations, in order to show the better rate-of-convergence stated in Theorem 3.1. To this end, in (3.4) we treat the second term from the left-hand-side as follows

$$
\int_{0}^{t} \int_{\Omega} \nabla u^{\nu} \nabla u d x d \tau=\int_{0}^{t} \int_{\Omega}|\nabla u|^{2} d x d \tau+\int_{0}^{t} \int_{\Omega} \nabla u^{E} \nabla u d x d \tau .
$$

We arrive now at [cf. (3.4)]

$$
\begin{aligned}
& \frac{\|u(t)\|^{2}}{2}+\nu \int_{0}^{t}\|\nabla u\|^{2} d \tau-\int_{0}^{t} \int_{\Omega}(u \cdot \nabla) u^{E} u d x d \tau+\nu \int_{0}^{t} \int_{\Gamma} u^{\nu}(\nabla n)^{T} u d S d \tau \\
& \leq-\nu \int_{0}^{t} \int_{\Omega} \nabla u^{E} \nabla u d x d \tau+\frac{\|u(0)\|^{2}}{2}
\end{aligned}
$$

We handle the third term from the left-hand-side exactly as before and, by integrating by parts the term from the right-hand-side (by using the second 
identity from Lemma 2.3), we get

$$
\begin{aligned}
-\nu \int_{0}^{t} \int_{\Omega} \nabla u^{E} \nabla u d x d \tau= & \nu \int_{0}^{t} \int_{\Gamma}\left(\omega^{E} \times n\right) u d S d \tau+\nu \int_{0}^{t} \int_{\Omega} \Delta u^{E} u d x d \tau \\
& +\nu \int_{0}^{t} \int_{\Gamma} u^{E} \cdot(\nabla n)^{T} \cdot u d S d \tau
\end{aligned}
$$

Then, we get the following inequality

$$
\begin{aligned}
\frac{\|u(t)\|^{2}}{2}+\nu \int_{0}^{t}\|\nabla u\|^{2} d \tau \leq & \frac{\|u(0)\|^{2}}{2}-\nu \int_{0}^{t} \int_{\Gamma} u(\nabla n)^{T} u d S d \tau \\
& -\int_{0}^{t} \int_{\Omega}(u \cdot \nabla) u^{E} u d x d \tau+\nu \int_{0}^{t} \int_{\Omega} \Delta u^{E} u d x d \tau \\
& -\nu \int_{0}^{t} \int_{\Gamma}\left(\omega^{E} \times n\right) u d S d \tau .
\end{aligned}
$$

We estimate the absolute value of the space integral from the right-handside by using Schwartz inequality, trace interpolation inequalities, and the regularity of $u^{E}$ as follows:

$$
\begin{aligned}
\nu\left|\int_{\Gamma}\left(\omega^{E} \times n\right) u d S\right| & \leq C \nu\|u\|^{\frac{1}{2}}\|\nabla u\|^{\frac{1}{2}} \leq C \nu^{\frac{3}{2}}+C\|u\|^{2}+\frac{\nu}{2}\|\nabla u\|^{2}, \\
\nu\left|\int_{\Gamma} u \cdot(\nabla n)^{T} \cdot u d S\right| & \leq C \nu\|u\|_{\Gamma}^{2} \leq C \nu\|u\|\|\nabla u\| \leq C \nu\|u\|^{2}+\frac{\nu}{2}\|\nabla u\|^{2}, \\
\nu\left|\int_{\Omega} \Delta u^{E} u d x\right| & \leq C\left(\|u\|^{2}+\nu^{2}\right) .
\end{aligned}
$$

Then, by using also the energy inequality, we obtain the following differential inequality [cf. with (3.5)]

$$
\|u(t)\|^{2}+\nu \int_{0}^{t}\|\nabla u(\tau)\|^{2} d \tau \leq\|u(0)\|^{2}+C\left[\int_{0}^{t}\|u(\tau)\|^{2} d \tau+\nu^{2}+\nu^{\frac{3}{2}}\right] .
$$

By using Gronwall-Lemma we have that

$$
\begin{aligned}
\left\|u^{\nu}-u^{E}\right\|_{L^{\infty}\left(0, T ; L^{2}(\Omega)\right)}^{2} & =O\left(\nu^{\frac{3}{2}}\right), \\
\left\|\nabla u^{\nu}-\nabla u^{E}\right\|_{L^{2}\left(0, T ; L^{2}(\Omega)\right)}^{2} & =O\left(\nu^{\frac{1}{2}}\right) .
\end{aligned}
$$

ending the proof.

We want now to show better convergence, and this happens if the initial datum belongs to a particular sub-class. In particular, we use the same observation made in $[8,32]$ to show strong convergence up to second order derivatives. We prove now the main result of the paper.

Theorem 3.2. Let $\Omega$ be a bounded smooth open set in $\mathbb{R}^{3}$, and let $u_{0}^{E} \in H^{3}(\Omega)$, be a divergence-free vector field tangential to the boundary, and such that

$$
\omega_{0}^{E}(x)=0 \quad \forall x \in \Gamma .
$$


Let $u^{E} \in C\left([0, T] ; H^{3}(\Omega)\right)$ be the unique solution of the Euler equations (1.2), with initial datum $u_{0}^{E}$ and defined in some interval $[0, T]$. Let $u^{\nu}$ be a weak solution of the Navier-Stokes equations (1.3) with a divergence free and tangential to the boundary initial datum $u_{0}^{n} \in L^{2}(\Omega)$ such that

$$
\left\|u_{0}^{\nu}-u_{0}^{E}\right\|=\mathcal{O}(\nu)
$$

Then,

$$
\begin{gathered}
\sup _{t \in[0, T]}\left\|u^{\nu}-u^{E}\right\|_{L^{\infty}\left(0, T ; L^{2}(\Omega)\right)}^{2}=O\left(\nu^{2}\right), \\
\left\|\nabla u^{\nu}-\nabla u^{E}\right\|_{L^{2}\left(0, T ; L^{2}(\Omega)\right)}^{2}=O(\nu) .
\end{gathered}
$$

A critical point in the proof is that of "having solution to the Euler equations with vanishing tangential component of the vorticity, as the NavierStokes equations", that is $\omega^{E} \times n=0$ on $\Gamma \times[0, T]$ : in this way one can better estimate the term $-\int_{\Omega} \Delta u^{E} u d x$ involved in the previous calculations.

In general the boundary condition for the vorticity cannot be enforced for the Euler equations. In addition, also if the initial datum is such that $\left(\omega_{0}^{E} \times n\right)_{\mid \Gamma}=0$ this is not enough to have the same boundary behavior for all positive times. As observed in [4], by using the vorticity equation,

$$
\omega_{t}^{E}+\left(u^{E} \cdot \nabla\right) \omega^{E}=\left(\omega^{E} \cdot \nabla\right) u^{E},
$$

by taking the exterior product with the normal unit vector on $\Gamma$, and finally by using that $\omega^{E} \times n=0$ implies that $\omega_{t}^{E} \times n=0$ on $\Gamma$, one has as a consequence that an extra-compatibility condition, generically false, should be satisfied by the initial velocity $u_{0}^{E}$. In particular, this implies that the Navier's type condition does not persist for positive time and hence excludes the chance of a vanishing-viscosity limit in topologies such that the vorticity $\omega^{E}$ has Sobolev traces at the boundary. On the other hand, by using the fact that for the Euler equations the vorticity is transported by the velocity $u^{E}$ and stretched by $\nabla u^{E}$, one can employ the well-known representation formula for classical solutions

$$
\omega^{E}(X(\alpha, t), t)=\nabla_{\alpha} X(\alpha, t) \omega^{E}(\alpha, 0),
$$

where the path-lines $X: \Omega \times[0, T] \rightarrow \Omega \subset \mathbb{R}^{3}$ solve the Cauchy problem

$$
\left\{\begin{array}{l}
\frac{d}{d t} X(\alpha, t)=u^{E}(X(\alpha, t), t) \\
X(\alpha, 0)=\alpha
\end{array}\right.
$$

for $t \in[0, T]$ and $\alpha \in \Omega$. Since $u^{E} \cdot n=0$ on the boundary, the path-lines starting on the boundary remain on the boundary for all positive times. The fundamental effect for our studies is the following: let be given $\bar{\alpha} \in \Gamma$, then $X(\bar{\alpha}, t) \in \Gamma$ for all $t \in[0, T]$ and consequently

$$
\omega^{E}(X(\bar{\alpha}, t), t) \times n=\left[\nabla_{\alpha} X(\bar{\alpha}, t) \omega^{E}(\bar{\alpha}, 0)\right] \times n, \quad \forall(\bar{\alpha}, t) \in \Gamma \times[0, T] .
$$

Being generically the matrix $\nabla_{\alpha} X(\bar{\alpha}, t)$ not a multiple of the identity, this implies that generically $\omega^{E}(X(\bar{\alpha}, t), t) \times n \neq 0$. In particular $\omega^{E} \times n$ may become non-zero, as soon as $\nabla_{a} X$ induces a rotation along any axis not parallel with the normal unit vector passing through $\bar{\alpha}$. 
On the other hand, this observation makes clear that it is possible to have another type of persistence for the vorticity, by suitably restricting the class of initial data. In fact, the same representation formula with the path-lines shows also the following result.

Lemma 3.3. Let $u^{E} \in C\left([0, T] ; H^{3}(\Omega)\right)$ be the unique solution of the Euler equations (1.2). If $\omega_{0}^{E}(x)=0$ for all $x \in \Gamma$, then

$$
\omega^{E}(x, t)=0 \quad \text { for all couples }(x, t) \in \Gamma \times[0, T] .
$$

Remark 3.4. The class of solutions with vanishing-vorticity of the boundary we will employ is in some sense optimal, since in [5] it is shown that if $\omega_{0} \cdot n \neq 0$, then there exists a time $\bar{T}>0$ such that the $\omega^{E} \times n \neq 0$ for $0<t \leq \bar{T}$. Moreover this class of initial data in non-empty, since smooth and divergencefree functions with compact support satisfy the assumptions.

Remark 3.5. In the case of the Euler equations with a non-zero external force $f^{E}$ the same approach shows that a formula similar to (3.8) holds true:

$$
\omega^{E}(X(\alpha, t), t)=\nabla_{\alpha} X(\alpha, t) \omega^{E}(\alpha, 0)+\int_{0}^{t} \operatorname{curl} f^{E}(X(\alpha, \sigma), \sigma) d \sigma,
$$

hence a sufficient condition to have persistence of the vanishing vorticity at the boundary is $\omega_{0}^{E}=\operatorname{curl} f^{E}=0$ on $\Gamma$.

This observation on the persistence of the boundary values for the vorticity points out that probably the " $\omega$-based" boundary conditions of Navier'stype are much better behaved (in the context of vanishing-viscosity) than the classical "D $(u)$-based" Navier's ones. The Navier's conditions as in (1.3) involve the symmetric part of the gradient. One can recall that the symmetric part of the gradient $\mathcal{D}\left(u^{E}\right):=\frac{\left[\nabla u^{E}+\left(\nabla u^{E}\right)^{T}\right]}{2}$ has the following evolution equation

$$
\frac{D \mathcal{D}\left(u^{E}\right)}{D t}+\mathcal{D}\left(u^{E}\right)^{2}+\mathcal{O}^{2}\left(u^{E}\right)=-H \pi^{E}
$$

where as usual $\frac{D}{D t}$ is the derivative along path-lines, $\mathcal{O}\left(u^{E}\right):=\frac{\left[\nabla u^{E}-\left(\nabla u^{E}\right)^{T}\right]}{2}$ is essentially the vorticity (since $\mathcal{O}\left(u^{E}\right) h=\frac{1}{2} \omega^{E} \times h$ for each vector $h$ ) and $H \pi^{E}$ is the Hessian of the pressure. It seems that (contrary to the results for $\omega^{E}$ or equivalently $\mathcal{O}$ ) the evolution of the matrix $\mathcal{D}\left(u^{E}\right)$ cannot be handled, since the pressure does not disappear and one cannot employ directly a transport/stretching argument. Hence, the problem related with vanishing-viscosity under the Navier's boundary conditions seems to require different tools, even if the friction parameter $\beta$ vanishes.

Proof of Theorem 3.2. We can improve a little bit on the rate of convergence of Theorem 3.1, by assuming that the initial datum is such that the vorticity vanishes as the boundary. In fact, we have seen that by assuming (3.7) we have $\omega^{E}(x, t)=0$ for all $(x, t) \in \Gamma \times[0, T]$. Writing again the same energy estimates we employed before to get (3.6), and by using

$$
-\nu \int_{0}^{t} \int_{\Omega} \nabla u^{E} \nabla u d x d \tau=\nu \int_{0}^{t} \int_{\Gamma} u^{E} \cdot(\nabla n)^{T} \cdot u d S d \tau+\nu \int_{0}^{t} \int_{\Omega} \Delta u^{E} u d x d \tau,
$$


(notice that now the boundary integral $\int_{\Gamma}\left(\omega^{E} \times n\right) u d S$ vanishes) we can re-do the same calculations as before starting from (3.6) to obtain now

$$
\|u(t)\|^{2}+\nu \int_{0}^{t}\|\nabla u\|^{2} d \tau \leq\|u(0)\|^{2}+C\left[\int_{0}^{t}\|u(\tau)\|^{2} d \tau+\nu^{2}\right] .
$$

Finally, by Gronwall's inequality we get

$$
\begin{gathered}
\sup _{t \in[0, T]}\left\|u^{\nu}-u^{E}\right\|_{L^{\infty}\left(0, T ; L^{2}(\Omega)\right)}^{2}=O\left(\nu^{2}\right), \\
\left\|\nabla u^{\nu}-\nabla u^{E}\right\|_{L^{2}\left(0, T ; L^{2}(\Omega)\right)}^{2}=O(\nu) .
\end{gathered}
$$

concluding the proof.

\section{Vanishing viscosity for the 3D Boussinesq equations}

In this section we pass to consider the Boussinesq system (1.5) and a first step concerns the existence of weak solutions. In particular, since the problem is without diffusion in the equation for the density, a proper notion of weak solution is needed. Observe that the $\rho^{\nu}$ entering the equations is not the density, but represents the small variations of density from the constant state, hence there is no need to assume $\rho^{\nu} \geq 0$.

Definition 4.1. We say that the pair $u^{\nu} \in L^{\infty}\left(0, T ; L^{2}(\Omega)\right) \cap L^{2}\left(0, T ; H^{1}(\Omega)\right)$ and $\rho^{\nu} \in L^{\infty}\left(0, T ; L^{2}(\Omega)\right)$ is weak solution of the Boussinesq equations (1.5) if the two following conditions hold:

$$
\begin{aligned}
& \int_{0}^{T} \int_{\Omega}\left(-v^{\nu} \phi_{t}-\rho^{\nu} \psi_{t}+\nu \nabla v^{\nu} \nabla \phi-\left(v^{\nu} \cdot \nabla\right) \phi v^{\nu}-\left(v^{\nu} \cdot \nabla\right) \psi \rho^{\nu}\right) d x d \tau \\
& \quad+\nu \int_{0}^{T} \int_{\Gamma} v^{\nu} \cdot(\nabla n)^{T} \cdot \phi d S d \tau=\int_{\Omega} v_{0}^{\nu} \phi(0)+\rho_{0}^{\nu} \psi(0) d x
\end{aligned}
$$

for all vector-fields $\phi \in C_{0}^{\infty}([0, T[\times \bar{\Omega})$ such that $\nabla \cdot \phi=0$ in $\Omega \times[0, T[$, and $\phi \cdot n=0$ on $\Gamma \times\left[0, T\left[\right.\right.$ and scalars $\psi \in C_{0}^{\infty}([0, T[\times \bar{\Omega})$.

In particular the resulting solution satisfies the equations in the sense of $\mathcal{D}^{\prime}((0, T) \times \Omega)$ and the following energy estimate

$$
\begin{aligned}
& \frac{\left\|v^{\nu}(t)\right\|^{2}+\left\|\rho^{\nu}(t)\right\|^{2}}{2}+\nu \int_{0}^{t}\left\|\nabla v^{\nu}\right\|^{2} d \tau+\nu \int_{0}^{t} \int_{\Gamma} v^{\nu} \cdot(\nabla n)^{T} \cdot v^{\nu} d S d \tau \\
& \quad \leq \frac{\left\|v_{0}^{\nu}\right\|^{2}+\left\|\rho_{0}^{\nu}\right\|^{2}}{2}
\end{aligned}
$$

is satisfied for all $t \in[0, T]$.

With this definition we have the following result.

Theorem 4.2. Let be given any positive $T>0, v_{0}^{\nu} \in L^{2}(\Omega)$, and $\rho_{0}^{\nu} \in L^{2}(\Omega)$, such that $v_{0}^{\nu}$ is divergence-free and such that $v_{0}^{\nu} \cdot n=0$ on $\Gamma$. Then, there exists at least a weak solution of the Boussinesq equations (1.5) on $[0, T]$.

Proof. By simplifying a procedure typical of compressible flows, as in Lions [18] and Feireisl $[11, \S 4]$, we first consider the following approximate system, with $\epsilon>0$ 


$$
\begin{array}{ll}
\partial_{t} v^{\nu, \epsilon}-\nu \Delta v^{\nu, \epsilon}+\left(v^{\nu, \epsilon} \cdot \nabla\right) v^{\nu, \epsilon}+\nabla p^{\nu, \epsilon}=-\rho^{\nu, \epsilon} e_{3} & \text { in } \Omega \times(0, T], \\
\partial_{t} \rho^{\nu, \epsilon}+\left(v^{\nu, \epsilon} \cdot \nabla\right) \rho^{\nu, \epsilon}=\epsilon \Delta \rho^{\nu, \epsilon} & \text { in } \Omega \times(0, T], \\
\nabla \cdot v^{\nu, \epsilon}=0 & \text { in } \Omega \times(0, T], \\
v^{\nu, \epsilon} \cdot n=0 & \text { on } \Gamma \times(0, T], \\
\operatorname{curl} v^{\nu, \epsilon} \times n=0 & \text { on } \Gamma \times(0, T], \\
n \cdot \nabla \rho^{\nu, \epsilon}=0 & \text { on } \Gamma \times(0, T], \\
v^{\nu, \epsilon}(0, x)=v_{0}^{\nu} & \text { in } \Omega, \\
\rho^{\nu, \epsilon}(0, x)=\rho_{0}^{\nu} & \text { in } \Omega .
\end{array}
$$

By standard techniques one can show that the above system has at least a weak solution

$$
v^{\nu, \epsilon}, \rho^{\nu, \epsilon} \in L^{\infty}\left(0, T ; L^{2}(\Omega)\right) \cap L^{2}\left(0, T ; H^{1}(\Omega)\right),
$$

satisfying the energy inequality

$$
\begin{aligned}
& \frac{\left\|v^{\nu, \epsilon}(t)\right\|^{2}+\left\|\rho^{\nu, \epsilon}(t)\right\|^{2}}{2}+\nu \int_{0}^{t}\left\|\nabla v^{\nu, \epsilon}\right\|^{2} d \tau+\epsilon \int_{0}^{t}\left\|\nabla \rho^{\nu, \epsilon}\right\|^{2} d \tau \\
& +\nu \int_{0}^{t} \int_{\Gamma} v^{\nu, \epsilon} \cdot(\nabla n)^{T} \cdot v^{\nu, \epsilon} d S d \tau \leq \frac{\left\|v_{0}^{\nu}\right\|^{2}+\left\|\rho_{0}^{\nu}\right\|^{2}}{2} .
\end{aligned}
$$

As $\epsilon$ goes to zero, (and by standard results of compactness) one can find subsequences such that

$$
\begin{array}{ll}
v^{\nu, \epsilon} \stackrel{*}{\rightarrow} v^{\nu} \text { and } \rho^{\nu, \epsilon} \stackrel{*}{\rightarrow} \rho^{\nu} & \text { in } L^{\infty}\left(0, T ; L^{2}\right) \\
v^{\nu, \epsilon} \rightarrow v^{\nu} & \text { in } L^{2}\left(0, T ; H^{1}\right) \\
\rho^{\nu, \epsilon} \rightarrow \rho^{\epsilon} & \text { in } L^{2}\left(0, T ; L^{2}\right) \\
v^{\nu, \epsilon} \rightarrow v^{\nu} & \text { in } L^{2}\left(0, T ; L^{2}\right),
\end{array}
$$

for some $v^{\nu} \in L^{\infty}\left(0, T ; L^{2}(\Omega)\right) \cap L^{2}\left(0, T ; H^{1}(\Omega)\right)$ and $\rho^{\nu} \in L^{\infty}\left(0, T ; L^{2}(\Omega)\right)$. By standard arguments it turns out that $\left(v^{\nu}, \rho^{\nu}\right)$ is a weak solution to (1.5). In particular, being $v^{\nu, \epsilon}$ strongly convergent, while $\rho^{\nu, \epsilon}$ is weakly convergent in $L^{2}\left(0, T ; L^{2}(\Omega)\right)$, one has

$$
\int_{0}^{T} \int_{\Omega}\left(v^{\nu, \epsilon} \cdot\right) \nabla \psi \rho^{\nu, \epsilon} d x d \tau \rightarrow \int_{0}^{T} \int_{\Omega}\left(v^{\nu} \cdot \nabla\right) \psi \rho^{\nu} d x d \tau
$$

and also

$$
\epsilon \int_{0}^{T} \int_{\Omega} \nabla \rho^{\nu, \epsilon} \nabla \psi d x d \tau \rightarrow 0
$$

due to the uniform bound for $\epsilon \int_{0}^{T} \int_{\Omega}\left|\nabla \rho^{\nu, \epsilon}\right|^{2} d x d \tau$. This implies that the $\left(v^{\nu}, \rho^{\nu}\right)$ is a weak solution in the sense of Definition 4.1 and in particular that energy inequality (4.2) is satisfied.

From the existence result we prove now the last result of this paper.

Theorem 4.3. Let $\Omega$ be a bounded smooth open set in $\mathbb{R}^{3}$, let $v_{0}^{E} \in H^{3}(\Omega)$, be a divergence-free vector field tangential to the boundary, let also $\rho_{0}^{E} \in H^{3}(\Omega)$. Let us suppose that the initial data satisfy the following conditions at the boundary:

$$
\begin{array}{cc}
\omega_{0}^{E}(x)=0 & \forall x \in \Gamma, \\
\nabla \rho_{0}^{E}(x)=0 & \forall x \in \Gamma .
\end{array}
$$


Let $u^{E}, \rho^{E} \in C\left([0, T] ; H^{3}(\Omega)\right)$ be the unique solution of the Euler Boussinesq equations (1.6), with initial datum $\left(v_{0}^{E}, \rho_{0}^{E}\right)$ and defined in some interval $[0, T]$.

Let $\left(v^{\nu}, \rho^{\nu}\right)$ be a weak solution of the Boussinesq equations (1.5) with a divergence free and tangential to the boundary initial datum $u_{0}^{n} \in L^{2}(\Omega)$ and with $\rho_{0}^{\nu} \in L^{2}(\Omega)$ such that

$$
\left\|u_{0}^{\nu}-u_{0}^{E}\right\|=\mathcal{O}(\nu) \quad \text { and } \quad\left\|\rho_{0}^{\nu}-\rho_{0}^{E}\right\|=\mathcal{O}(\nu) .
$$

Then,

$$
\begin{gathered}
\sup _{t \in[0, T]}\left\|u^{\nu}-u^{E}\right\|_{L^{\infty}\left(0, T ; L^{2}(\Omega)\right)}^{2}=O\left(\nu^{2}\right), \\
\sup _{t \in[0, T]}\left\|\rho^{\nu}-\rho^{E}\right\|_{L^{\infty}\left(0, T ; L^{2}(\Omega)\right)}^{2}=O\left(\nu^{2}\right), \\
\left\|\nabla u^{\nu}-\nabla u^{E}\right\|_{L^{2}\left(0, T ; L^{2}(\Omega)\right)}^{2}=O(\nu) .
\end{gathered}
$$

Proof. The proof is based on the same techniques employed before. We multiply (1.5) by $\left(v^{E}, \rho^{E}\right)$ and with suitable integration by parts we get

$$
\begin{aligned}
& \int_{\Omega} v^{\nu}(t) u^{E}(t)+\rho^{\nu}(t) \rho^{E}(t) d x+\int_{0}^{t} \int_{\Omega}\left(\nu \nabla v^{\nu} \nabla v^{E}+\left(v^{\nu} \cdot \nabla\right) v^{\nu} v^{E}\right. \\
& \left.+\left(v^{\nu} \cdot \nabla\right) \rho^{\nu} \rho^{E}\right) d x d \tau-\int_{0}^{t} \int_{\Omega} \rho^{\nu} \rho_{t}^{E}+v^{\nu} v_{t}^{E} d x d \tau+\nu \int_{0}^{t} \int_{\Gamma} v^{\nu}(\nabla n)^{T} v^{E} d S d \tau \\
& \quad=\int_{\Omega} v_{0}^{E} v_{0}^{\nu}+\rho_{0}^{E} \rho_{0}^{\nu} d x
\end{aligned}
$$

We then obtain from the equation for $\left(v^{E}, \rho^{E}\right)$

$$
\begin{aligned}
\int_{0}^{t} & \int_{\Omega}\left(v_{t}^{E} v^{\nu}+\rho_{t}^{E} \rho^{\nu}+\left(v^{E} \cdot \nabla\right) v^{E} v^{\nu}+\left(v^{E} \cdot \nabla\right) \rho^{E} v^{\nu}\right) d x d \tau \\
& =\int_{\Omega} v_{0}^{E} v_{0}^{\nu}+\rho_{0}^{E} \rho_{0}^{\nu} d x
\end{aligned}
$$

Next, by multiplying the Euler Boussinesq equations by $u^{E}$ and by the usual integrations by parts we get the energy conservation

$$
\frac{\left\|v^{E}(t)\right\|^{2}+\left\|\rho^{E}(t)\right\|^{2}}{2}=\frac{\left\|v_{0}^{E}\right\|^{2}+\left\|\rho_{0}^{E}\right\|^{2}}{2} .
$$

Then, by adding together (4.2)-(4.6) and subtracting (4.4)-(4.5), we get

$$
\begin{aligned}
& \frac{\|v(t)\|^{2}+\|\rho(t)\|^{2}}{2}+\nu \int_{0}^{t} \int_{\Omega} \nabla v^{\nu} \nabla v d x d \tau+\int_{0}^{t} \int_{\Omega}(v \cdot \nabla) v^{E} v d x d \tau \\
& -\int_{0}^{t} \int_{\Omega}(v \cdot \nabla) \rho^{E} \rho d x d \tau+\nu \int_{0}^{t} \int_{\Gamma} v^{\nu}(\nabla n)^{T} v d S d \tau \leq \frac{\|v(0)\|+\|\rho(0)\|^{2}}{2}
\end{aligned}
$$

where

$$
v:=v^{\nu}-v^{E} \quad \text { and } \quad \rho:=\rho^{\nu}-\rho^{E} .
$$

With the same manipulations employed in the previous section we get 


$$
\begin{aligned}
& \frac{\|v(t)\|^{2}+\|\rho(t)\|^{2}}{2}+\nu \int_{0}^{t}\|\nabla v\|^{2} d \tau \\
& \leq \frac{\|v(0)\|^{2}+\|\rho(0)\|^{2}}{2}-\nu \int_{0}^{t} \int_{\Gamma} v(\nabla n)^{T} v d S d \tau-\int_{0}^{t} \int_{\Omega}(v \cdot \nabla) v^{E} v \\
& \quad+(v \cdot \nabla) \rho^{E} \rho d x d \tau-\nu \int_{0}^{t} \int_{\Omega} \Delta v^{E} v d x d \tau+\nu \int_{0}^{t} \int_{\Gamma}\left(\operatorname{curl} v^{E} \times n\right) v d S d \tau .
\end{aligned}
$$

We then estimate most of the terms as before with

$$
\begin{aligned}
\left|\int_{\Omega}(v \cdot \nabla) v^{E} v+(v \cdot \nabla) \rho^{E} \rho d x\right| & \leq C\left(\|u(t)\|^{2}+\|\rho(t)\|^{2}\right), \\
\nu\left|\int_{\Gamma} v \cdot(\nabla n)^{T} \cdot v d S\right| & \leq C \nu\|v\|^{2}+\frac{\nu}{2}\|\nabla v\|^{2}, \\
\nu\left|\int_{\Omega} \Delta v^{E} v d x\right| & \leq C\left(\|v\|^{2}+\nu^{2}\right),
\end{aligned}
$$

where we used that $v^{E} \in C\left([0, T] ; H^{3}(\Omega)\right)$.

To handle the last integral involving $\operatorname{curl} v^{E}$ on $\Gamma$, we observe that the equation for the vorticity for the Euler-Boussinesq system implies, along pathlines,

$\operatorname{curl} v^{E}(X(\alpha, t), t)=\nabla_{\alpha} X(\alpha, t) \operatorname{curl} v^{E}(\alpha, 0)-\int_{0}^{t} \operatorname{curl}\left(\rho^{E} e_{3}\right)(X(\alpha, \sigma), \sigma) d \sigma$,

hence now $\operatorname{curl} v_{0}^{E}(x)=0$ on $\Gamma$ is not enough to have $\operatorname{curl} v^{E}=0$ on the boundary for all positive times and a control also on $\operatorname{curl}\left(\rho^{E} e_{3}\right)$ at the boundary is needed. We then observe that $\rho^{E}$ is transported by the velocity $v^{E}$ (it solves the continuity equation), hence

$$
\rho^{E}(X(\alpha, t), t)=\rho_{0}^{E}(\alpha) .
$$

Consequently, we get the following evolution equation for the gradient of $\rho$ :

$$
\nabla \rho^{E}(X(\alpha, t), t) \nabla_{\alpha} X(\alpha, t)=\nabla \rho_{0}^{E}(\alpha)
$$

hence

$$
\nabla \rho^{E}(X(\alpha, t), t)=\nabla \rho_{0}^{E}(\alpha)\left[\nabla_{\alpha} X(\alpha, t)\right]^{-1} .
$$

Since the matrix $\nabla_{\alpha} X$ is non-singular and since path-lines starting at the boundary remain at the boundary, it follows that a sufficient condition to have $\operatorname{curl}\left(\rho^{E} e_{3}\right)(x, t)=0$ for all $x \in \Gamma$ and for all $t \in[0, T]$ is that of asking (4.3). Under the above assumptions the term $\int_{\Gamma}\left(\operatorname{curl} v^{E} \times n\right) v d S$ vanishes identically, hence we arrive at the inequality

$$
\begin{aligned}
& \|u(t)\|^{2}+\|\rho(t)\|^{2}+\nu \int_{0}^{t}\|\nabla u\|^{2} d \tau \leq\|u(0)\|^{2}+\|\rho(0)\|^{2} \\
& \quad+C\left[\int_{0}^{t}\|u(\tau)\|^{2} d \tau+\nu^{2}\right]
\end{aligned}
$$

from which we have the thesis by applying the Gronwall lemma. 


\section{References}

[1] Bardos, C.: Existence et unicité de la solution de l'équation d'Euler en dimension deux. J. Math. Anal. Appl. 40, 769-790 (1972)

[2] Beirão da Veiga, H., Berselli, L.C.: Navier-Stokes equations: Green's matrices, vorticity direction, and regularity up to the boundary. J. Differ. Equ. 246(2), 597$628(2009)$

[3] Beirão da Veiga, H., Crispo, F.: Concerning the $W^{k, p}$-inviscid limit for 3-D flows under a slip boundary condition. J. Math. Fluid Mech. 13(1), 117-135 (2011)

[4] Beirão da Veiga, H., Crispo, F.: The 3-d inviscid limit result under slip boundary conditions. A negative answer. J. Math. Fluid Mech. 14(1), 55-59 (2012)

[5] Beirão da Veiga, H., Crispo, F.: A missed persistence property for the Euler equations, and its effect on inviscid limits. Nonlinearity 25, 1661-1669 (2012)

[6] Beirão da Veiga, H., Crispo, F.: Sharp inviscid limit results under Navier type boundary conditions. An $L^{p}$ theory. J. Math. Fluid Mech. 12, 397-411 (2010)

[7] Berselli, L.C.: Some results on the Navier-Stokes equations with Navier boundary conditions, 2010. Riv. Mat. Univ. Parma. 1, 1-75 (Lecture notes of a course given at SISSA/ISAS, Trieste, Sept 2009) (2010)

[8] Berselli, L.C., Spirito, S.: On the vanishing viscosity limit for the Navier-Stokes equations under slip boundary conditions in general domains. Commun. Math. Phys. 316(1), 171-198 (2012)

[9] Constantin, P.: On the Euler equations of incompressible fluids. Bull. Am. Math. Soc. (N.S.) 44(4), 603-621 (2007)

[10] Ebin, D., Marsden, J.E.: Groups of diffeomorphisms and the motion of an incompressible fluid. Ann. Math. 93, 102-63 (1970)

[11] Feireisl, E.: Dynamics of viscous compressible fluids, vol. 26 of Oxford Lecture Series in Mathematics and its Applications. Oxford University Press, Oxford (2004)

[12] Iftimie, D., Planas, G.: Inviscid limits for the Navier-Stokes equations with Navier friction boundary conditions. Nonlinearity 19(4), 899-918 (2006)

[13] Iftimie, D., Sueur, F.: Viscous boundary layers for the Navier-Stokes equations with the Navier slip conditions. Arch. Ration. Mech. Anal. 199(1), 145175 (2011)

[14] Kato, T.: Remarks on zero viscosity limit for nonstationary Navier-Stokes flows with boundary. In: Chern, S.S. (ed.) Seminar on Nonlinear Partial Differential Equations (Berkeley, California, 1983), vol. 2 of Math. Sci. Res. Inst. Publ., pp. 85-98. Springer, New York (1984)

[15] Kelliher, J.P.: On Kato's conditions for vanishing viscosity. Indiana Univ. Math. J. 56(4), 1711-1721 (2007) 
[16] Kozono, H., Yanagisawa, T.: $L^{r}$-variational inequality for vector fields and the Helmholtz-Weyl decomposition in bounded domains. Indiana Univ. Math. J. 58(4), 1853-1920 (2009)

[17] Lions, J.-L.: Quelques méthodes de résolution des problèmes aux limites non linéaires. Dunod, Gauthier-Villars, Paris (1969)

[18] Lions, P.-L.: Mathematical topics in fluid mechanics. Vol. 1, volume 3 of Oxford Lecture Series in Mathematics and its Applications. The Clarendon Press Oxford University Press, New York. Incompressible models, Oxford Science Publications, Oxford (1996)

[19] Lopes Filho, M.C., Mazzucato, A., Nussenzveig Lopes, H.J.: Vanishing viscosity limit for the incompressible flow inside a rotating circle. Phys. D 237(10-12), 1324-1333 (2008)

[20] Lopes Filho, M.C., Nussenzveig Lopes, H.J., Planas, G.: On the inviscid limit for two-dimensional incompressible flow with Navier friction condition. SIAM J. Math. Anal. 36, 1130-1141 (2005)

[21] Masmoudi, N., Rousset, F.: Uniform regularity for the Navier-Stokes equation with Navier boundary condition. Arch. Ration. Mech. Anal. 203(2), 529$575(2012)$

[22] Maxwell, J.C.: On stresses in rarefied gases arising from inequalities of temperature. Philos. Trans. R. Soc. Lond. 170, 249-256 (1879)

[23] Mazzucato, A.: On the zero viscosity limit in incompressible fluids. Phys. Scr. 0140002, 6 (2008)

[24] Navier, C.L.M.H.: Mémoire sur les Lois du Mouvement des Fluides. Mém. Acad. Sci. Inst. Fr. (2) 6, 389-440 (1823)

[25] Solonnikov, V.A., Ščadilov, V.E.: A certain boundary value problem for the stationary system of Navier-Stokes equations. Trudy Mat. Inst. Steklov. 125, 196-210, 235. Boundary value problems of mathematical physics, 8 (1973)

[26] Temam, R.: On the Euler equations of incompressible perfect fluids. J. Funct. Anal. 20(1), 32-43 (1975)

[27] Temam, R., Wang, X.: On the behavior of the solutions of the Navier-Stokes equations at vanishing viscosity. Ann. Scuola Norm. Sup. Pisa Cl. Sci. (4) 25(34), 807-828 (1998), 1997. Dedicated to Ennio De Giorgi

[28] Wang, X.: A Kato type theorem on zero viscosity limit of Navier-Stokes flows. Indiana Univ. Math. J. 50(Special Issue), 223-241 (2001). Dedicated to Professors Ciprian Foias and Roger Temam (Bloomington, IN, 2000)

[29] Wang, X., Wang, Y.-G., Xin, Z.: Boundary layers in incompressible NavierStokes equations with Navier boundary conditions for the vanishing viscosity limit. Commun. Math. Sci. 8(4), 965-998 (2010)

[30] Wang, Y.-G., Xin, Z.: Zero viscosity limit for incompressible Navier-Stokes equations with the Navier boundary conditions. Technical Report (2009) 
[31] Xiao, Y., Xin, Z.: On the vanishing viscosity limit for the 3D Navier-Stokes equations with a slip boundary condition. Commun. Pure Appl. Math. 60(7), 10271055 (2007)

[32] Xiao, Y., Xin, Z.: Remarks on vanishing viscosity limits for the 3D Navier-Stokes equations with a slip boundary condition. Chin. Ann. Math. 32B(3), 321-332 (2011)

[33] Xiao, Y., Xin, Z., Wu, J.: Vanishing viscosity limit for the 3D magnetohydrodynamic system with a slip boundary condition. J. Funct. Anal. 257(11), 33753394 (2009)

[34] Yudovich, V.I.: Non stationary flow of an ideal incompressible liquid (Russian). Comput. Math. Math. Phys. 3, 1407-1456 (1963)

Luigi C. Berselli

Dipartimento di Matematica

Università degli Studi di Pisa

Via F. Buonarroti 1/c

56127 Pisa

Italy

e-mail: berselli@dma.unipi.it

Stefano Spirito

Department Matematik und Informatik

Universität Basel

Rheinsprung 21

4051 Basel

Switzerland

e-mail: stefano.spirito@unibas.ch

Received: 4 February 2013.

Accepted: 7 July 2013. 